\title{
Harmonisierung bleibt auf der Agenda
}

\section{Liebe Leserinnen und Leser,}

äußert man die Meinung, dass die Wissenschaft der Praxis stets geraume Zeit voraus ist, erntet man zumeist keinen großen Widerspruch. Gehen wir einmal davon aus, dass dies nicht nur für die Natur- und Ingenieurwissenschaften gilt, sondern auch für die Betriebswirtschaftslehre. Dann sieht es um das interne Rechnungswesen, die Bastion der Controller, nicht sehr gut aus. Blicken wir in die USA als Hort des betriebswirtschaftlichen Fortschritts: Dort ist es an den TopUniversitäten längst so, dass das Financial Accounting eine deutlich höhere Bedeutung als das Management Accounting besitzt. Dies wird in der Zukunft eher noch zu- als abnehmen: Grund hierfür ist die Anreizstruktur der nachrückenden jungen Wissenschaftler. Sie gehen im Accounting dorthin, wo sie die größten Chancen haben, publizieren zu können und eine Professur zu erhalten - also ins Financial Accounting.

In Deutschland hat sich lange Zeit eine eher umgekehrte Entwicklung gezeigt. In zwei Jahrzehnten wuchs die Zahl der Controlling-Lehrstühle auf über 70 an. Mit der Internationalisierung des externen Rechnungswesens und der Internationalisierung der deutschen betriebswirtschaftlichen Forschung ist nun aber der gegenläufige Trend zu beobachten: Erste Hochschulen wandeln Controlling-Lehrstühle in solche für externes Rechnungswesen um, und auch im Curriculum mancher Master- und MBA-Programme hat das Management Accounting bzw. das Controlling gegenüber dem Financial Accounting einen zunehmend schweren Stand.

In den Unternehmen war der Einzug von Daten der externen Rechnungslegung in die interne Steuerung anfangs ein Thema von Großunternehmen. Es war zudem unklar, was das für die Beziehungen zwischen Rechnungswesen und Controlling bedeuten würde. In der Folge begann ein Konkurrenzkampf um die Aufmerksamkeit des Managements. Heute ist die Situation weitgehend entspannt. Die Vorteile einer Harmonisierung werden im Wesentlichen genutzt. Sie beschränkt sich zumeist auf die Ebene des Gesamtunternehmens, die Werke werden häufig noch mit internen Erfolgsdaten geführt. Insgesamt ist das Thema damit als im Grundsatz entschieden bzw. umgesetzt zu sehen. Bei unserer Erhebung der zehn



Utz Schäffer

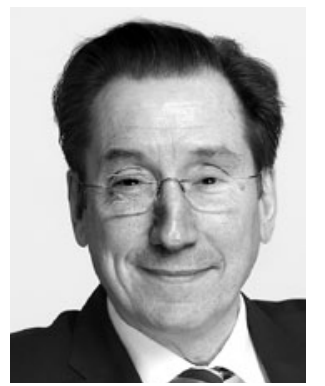

Jürgen Weber
Zukunftsthemen des Controllings liegt die Harmonisierung entsprechend abgeschlagen auf dem letzten Platz. Dennoch: Auch auf der Ebene von Großunternehmen haben einige noch viele Schritte des Wegs der Harmonisierung vor sich (vgl. z. B. das Interview mit ThyssenKrupp in diesem Heft), und das Thema ist nie abgeschlossen. Bei jeder Veränderung der Vorschriften der externen Rechnungslegung ist zu fragen, was das für die internen Steuerungsgrößen bedeutet.

Wer diese Fragen stellt und beantwortet, ist durchaus noch offen. Wer die Referenz der akademischen Welt heranzieht, hat hierfür eine klare Antwort parat. Controller sollten sich dieser bewusst sein und durch ihre Steuerungskompetenz ihre Position halten. In den Schoß fallen wird ihnen das Thema Harmonisierung aber nicht!

Viel Spaß bei der Lektüre wünschen Ihnen
